Proyecciones Journal of Mathematics

Vol. 36, No 3, pp. 373-395, September 2017.

Universidad Católica del Norte

Antofagasta - Chile

\title{
On some generalized geometric difference sequence spaces
}

\author{
Khirod Boruah \\ Rajiv Gandhi University, India \\ and \\ Bipan Hazarika \\ Rajiv Gandhi University, India \\ Received: October 2016. Accepted : April 2017
}

\begin{abstract}
In this paper we introduce the generalized geometric difference sequence spaces $\ell_{\infty}^{G}\left(\Delta_{G}^{m}\right), c^{G}\left(\Delta_{G}^{m}\right), c_{0}^{G}\left(\Delta_{G}^{m}\right)$, and to prove that these are Banach spaces. Then we prove some inclusion properties. Also we compute their dual spaces.
\end{abstract}

Keywords and phrases : Geometric difference; dual space; geometric integers; geometric real numbers.

AMS subject classification (2000) : 26A06, 11U10, 08A05, 46A45. 


\section{Introduction}

In the area of non-Newtonian calculus pioneering work was carried out by Grossman and Katz [9] which we call as multiplicative calculus. The operations of multiplicative calculus are called as multiplicative derivative and multiplicative integral. We refer to M. Grossman [10], Grossman et al. [11], Jane Grossman [12], Stanley [17], Bashirov et al. [1, 2] for different types of Non-Newtonian calculi and its applications. An extension of multiplicative calculus to functions of complex variables is handled by Bashirov and Riza [3], Uzer [20], Çakmak and Başar [5], Tekin and Başar[18], Türkmen and Başar [19].

Nowadays geometric calculus is an alternative to the usual calculus of Newton and Leibniz. It provides differentiation and integration tools based on multiplication instead of addition. Almost all properties in Newtonian calculus has an analog in multiplicative calculus. Generally speaking multiplicative calculus is a methodology that allows one to have a different look at problems which can be investigated via calculus. In some cases, mainly problems of price elasticity, multiplicative growth etc. the use of multiplicative calculus is advocated instead of a traditional Newtonian one. To know better about Non-Newtonian calculus, we must have idea about different types of arithmetics and their generators.

\section{2. $\alpha$-generator and geometric real field}

A generator is a one-to-one function whose domain is $\mathbf{R}$ (the set of real numbers) and range is a set $A \subset \mathbf{R}$. Each generator generates exactly one arithmetic and each arithmetic is generated by exactly one generator. For example, the identity function generates classical arithmetic, and exponential function generates geometric arithmetic. As a generator, we choose the function $\alpha$ such that whose basic algebraic operations are defined as follows:

$$
\begin{aligned}
& \alpha-\operatorname{addition} x \dot{+} y=\alpha\left[\alpha^{-1}(x)+\alpha^{-1}(y)\right] \\
& \alpha-\operatorname{subtraction} x \dot{-} y=\alpha\left[\alpha^{-1}(x)-\alpha^{-1}(y)\right] \\
& \alpha-\text { multiplication } x \dot{\times} y=\alpha\left[\alpha^{-1}(x) \times \alpha^{-1}(y)\right] \\
& \alpha-\operatorname{division} x / y=\alpha\left[\alpha^{-1}(x) / \alpha^{-1}(y)\right] \\
& \alpha-\operatorname{order} x \dot{<} y \Leftrightarrow \alpha^{-1}(x)<\alpha^{-1}(y) .
\end{aligned}
$$

for $x, y \in A$, where $A$ is a range of the function $\alpha$.

If we choose $\exp$ as an $\alpha$-generator defined by $\alpha(z)=e^{z}$ for $z \in \mathbf{R}$ 
then $\alpha^{-1}(z)=\ln z$ and $\alpha$-arithmetic turns out to geometric arithmetic.

$$
\begin{aligned}
& \alpha-\operatorname{addition} x \oplus y=\alpha\left[\alpha^{-1}(x)+\alpha^{-1}(y)\right]=e^{(\ln x+\ln y)}=x . y \\
& \text { geometric addition } \\
& \alpha-\operatorname{subtraction} x \ominus y=\alpha\left[\alpha^{-1}(x)-\alpha^{-1}(y)\right]=e^{(\ln x-\ln y)}=x \div y, y \neq 0 \\
& \text { geometric subtraction } \\
& \alpha-\text { multiplication } x \odot y=\alpha\left[\alpha^{-1}(x) \times \alpha^{-1}(y)\right]=e^{(\ln x \times \ln y)}=x^{\ln y} \\
& \text { geometric multiplication } \\
& \alpha-\operatorname{division} x \oslash y=\alpha\left[\alpha^{-1}(x) / \alpha^{-1}(y)\right]=e^{(\ln x \div \ln y)}=x^{\frac{1}{\ln y}}, y \neq 1 \\
& \text { geometric division. }
\end{aligned}
$$

It is obvious that $\ln (x)<\ln (y)$ if $x<y$ for $x, y \in \mathbf{R}^{+}$. That is, $x<$ $y \Leftrightarrow \alpha^{-1}(x)<\alpha^{-1}(y)$ So, without loss of generality, we use $x<y$ instead of the geometric order $x \dot{<} y$.

C. Türkmen and F. Başar [19] defined the sets of geometric integers, geometric real numbers and geometric complex numbers $\mathbf{Z}(G), \mathbf{R}(G)$ and $\mathbf{C}(G)$, respectively, as follows:

$$
\begin{array}{lcc}
\mathbf{Z}(G)= & \left\{e^{x}: x \in \mathbf{Z}\right\} \\
\mathbf{R}(G)= & \left\{e^{x}: x \in \mathbf{R}\right\}=\mathbf{R}^{+} \backslash\{0\} \\
\mathbf{C}(G)= & \left\{e^{z}: z \in \mathbf{C}\right\}=\mathbf{C} \backslash\{0\} .
\end{array}
$$

Remark 2.1. $(\mathbf{R}(G), \oplus, \odot)$ is a field with geometric zero 1 and geometric identity $e$, since

(1). $(\mathbf{R}(G), \oplus)$ is a geometric additive Abelian group with geometric zero 1 ,

(2). $(\mathbf{R}(G) \backslash 1, \odot)$ is a geometric multiplicative Abelian group with geometric identity $e$,

(3). $\odot$ is distributive over $\oplus$.

But $(\mathbf{C}(G), \oplus, \odot)$ is not a field, however, geometric binary operation $\odot$ is not associative in $\mathbf{C}(G)$. For, we take $x=e^{1 / 4}, y=e^{4}$ and $z=e^{(1+i \pi / 2)}=$ ie. Then

$$
\begin{array}{ccc}
(x \odot y) \odot z & =e \odot z=z=i e \\
\text { but } x \odot(y \odot z) & =x \odot e^{4}=e .
\end{array}
$$


Let us define geometric positive real numbers and geometric negative real numbers as follows:

$$
\begin{aligned}
& \mathbf{R}^{+}(G)=\{x \in \mathbf{R}(G): x>1\} \\
& \mathbf{R}^{-}(G)=\{x \in \mathbf{R}(G): x<1\} .
\end{aligned}
$$

Then for all $x, y \in \mathbf{R}(G)$

- $x \oplus y=x y$

- $x \ominus y=x / y$

- $x \odot y=x^{\ln y}=y^{\ln x}$

- $x \oslash y$ or $\frac{x}{y} G=x^{\frac{1}{\ln y}}, y \neq 1$

- $x_{1} \oplus x_{2} \oplus \ldots \oplus x_{n}={ }_{G} \sum_{i=1}^{n} x_{i}=x_{1} . x_{2} \ldots x_{n}$

- $x^{2_{G}}=x \odot x=x^{\ln x}$

- $x^{p_{G}}=x^{\ln ^{p-1} x}$

- $\sqrt{x}^{G}=e^{(\ln x)^{\frac{1}{2}}}$

- $x^{-1_{G}}=e^{\frac{1}{\log x}}$

- $x \odot e=x$ and $x \oplus 1=x$

- $e^{n} \odot x=x^{n}=x \oplus x \oplus$.....(upto $n$ number of $x$ )

$$
|x|^{G}= \begin{cases}x, & \text { if } x>1 \\ 1, & \text { if } x=1 \\ \frac{1}{x}, & \text { if } x<1\end{cases}
$$

- Thus $|x|^{G} \geq 1$.

- ${\sqrt{x^{2}}}^{G}=|x|^{G}$

- $\left|e^{y}\right|^{G}=e^{|y|}$

- $|x \odot y|^{G}=|x|^{G} \odot|y|^{G}$

- $|x \oplus y|^{G} \leq|x|^{G} \oplus|y|^{G}$

- $|x \oslash y|^{G}=|x|^{G} \oslash|y|^{G}$

- $|x \ominus y|^{G} \geq|x|^{G} \ominus|y|^{G}$

- $0_{G} \ominus 1_{G} \odot(x \ominus y)=y \ominus x$, i.e. in short $\ominus(x \ominus y)=y \ominus x$. 


\subsection{Geometric Limit}

According to Grossman and Katz [9], geometric limit of a positive valued function defined in a positive interval is same to the ordinary limit. Here, we define Geometric limit of a function with the help of geometric arithmetic as follows:

A function $f$, which is positive in a given positive interval, is said to tend to the limit $l>0$ as $x$ tends to $a \in \mathbf{R}$, if, corresponding to any arbitrarily chosen number $\epsilon>1$, however small(but greater than 1), there exists a positive number $\delta>1$, such that

$$
1<|f(x) \ominus l|^{G}<\epsilon
$$

for all values of $x$ for which $1<|x \ominus a|^{G}<\delta$. We write

$$
\lim _{x \rightarrow a} f(x)=l \text { or } f(x) G l .
$$

Here,

$$
\begin{aligned}
|x \ominus a|^{G}<\delta & \Rightarrow\left|\frac{x}{a}\right|^{G}<\delta \\
& \Rightarrow \frac{1}{\delta}<\frac{x}{a}<\delta \\
& \Rightarrow \frac{a}{\delta}<x<a \delta .
\end{aligned}
$$

Similarly, $|f(x) \ominus l|^{G}<\epsilon \Rightarrow \frac{l}{\epsilon}<f(x)<l \epsilon$.

Thus, $f(x) G l$ means that for any given positive real number $\epsilon>1$, no matter however closer to $1, \exists$ a finite number $\delta>1$ such that $f(x) \in] \frac{l}{\epsilon}, l \epsilon[$ for every $x \in] \frac{a}{\delta}, a \delta[$. It is to be noted that lengths of the open intervals $] \frac{a}{\delta}, a \delta[$ and $] \frac{l}{\epsilon}, l \epsilon$ [ decreases as $\delta$ and $\epsilon$ respectively decreases to 1 . Therefore, as $\epsilon$ decreases to $1, f(x)$ becomes closer and closer to $l$, as well as $x$ becomes closer and closer to $a$ as $\delta$ decreases to 1 . Hence, $l$ is also the ordinary limit of $f(x)$. i.e. $f(x) G l \Rightarrow f(x) \rightarrow l$. In other words, we can say that G-limit and ordinary limit are same for bipositive functions whose functional values as well as arguments are positive in the given interval. Only difference is that in geometric calculus we approach the limit geometrically, but in ordinary calculus we approach the limit linearly.

A function $f$ is said to tend to limit $l$ as $x$ tends to $a$ from the left, if for each $\epsilon>1$ (however small), there exists $\delta>1$ such that $|f(x) \ominus l|^{G}<\epsilon$ when $a / \delta<x<a$. In symbols, we then write

$$
G \lim _{x \rightarrow a-} f(x)=l \text { or } f(a-1)=l .
$$

Similarly, a function $f$ is said to tend to limit $l$ as $x$ tends to $a$ from the right, if for each $\epsilon>1$ (however small), there exists $\delta>1$ such that 
$|f(x) \ominus l|^{G}<\epsilon$ when $a<x<a \delta$. In symbols, we then write

$$
G \lim _{x \rightarrow a+} f(x)=l \text { or } f(a+1)=l .
$$

If $f(x)$ is negative valued in a given interval, it will be said to tend to a limit $l<0$ if for $\epsilon>1, \exists \delta>1$ such that $f(x) \in] l \epsilon, \frac{l}{\epsilon}[$ whenever $x \in] \frac{a}{\delta}, a \delta[$.

\subsection{Geometric Continuity}

A function $f$ is said to be geometric continuous at $x=a$ if

(i) $f(a)$ i.e., the value of $f(x)$ at $x=a$, is a definite number,

(ii) the Geometric-limit of the function $f(x)$ as $x G a$ exists and is equal to $f(a)$.

Alternatively, a function $f$ is said to be Geometric-continuous at $x=a$, if for arbitrarily chosen $\epsilon>1$, however small, there exists a number $\delta>1$ such that

$$
|f(x) \ominus f(a)|^{G}<\epsilon
$$

for all values of $x$ for which, $|x \ominus a|^{G}<\delta$.

On comparing the above definitions of limits and continuity, we can conclude that a function $f$ is geometric-continuous at $x=a$ if

$$
\lim _{x \rightarrow a} \frac{f(x)}{f(a)}=1 .
$$

Let $\ell_{\infty}, c$ and $c_{0}$ be the linear spaces of complex bounded, convergent and null sequences, respectively, normed by

$$
\|x\|_{\infty}=\sup _{k}\left|x_{k}\right|
$$

It is easy to prove that

$$
\omega(G)=\left\{\left(x_{k}\right): x_{k} \in \mathbf{R}(G) \text { for all } k \in \mathbf{N}\right\}
$$


is a vector space over $\mathbf{R}(G)$ with respect to the algebraic operations $\oplus$ addition and $\odot$ multiplication

$$
\begin{gathered}
\oplus: \omega(G) \times \omega(G) \rightarrow \omega(G) \\
(x, y) \rightarrow x \oplus y=\left(x_{k}\right) \oplus\left(y_{k}\right)=\left(x_{k} y_{k}\right) \\
\odot: \mathbf{R}(\mathbf{G}) \times \omega(G) \rightarrow \omega(G) \\
(\alpha, y) \rightarrow \alpha \odot y=\alpha \odot\left(y_{k}\right)=\left(\alpha^{\ln y_{k}}\right),
\end{gathered}
$$

where $x=\left(x_{k}\right), y=\left(y_{k}\right) \in \omega(G)$ and $\alpha \in \mathbf{R}(G)$. Then

$$
\begin{gathered}
\ell_{\infty}(G)=\left\{x=\left(x_{k}\right) \in \omega(G): \sup _{k \in \mathbf{N}}\left|x_{k}\right|^{G}<\infty\right\} \\
c(G)=\left\{x=\left(x_{k}\right) \in \omega(G): G \lim _{k \rightarrow \infty}\left|x_{k} \ominus l\right|^{G}=1\right\} \\
c_{0}(G)=\left\{x=\left(x_{k}\right) \in \omega(G): \lim _{k \rightarrow \infty} x_{k}=1\right\} \\
\ell_{p}(G)=\left\{x=\left(x_{k}\right) \in \omega(G): G \sum_{k=0}^{\infty}\left(\left|x_{k}\right|^{G}\right)^{p_{G}}<\infty\right\},
\end{gathered}
$$

where $G \sum$ is the geometric sum,

are classical sequence spaces over the field $\mathbf{R}(G)$. Also they have shown that $\ell_{\infty}(G), c(G)$ and $c_{0}(G)$ are Banach spaces with the norm

$$
\|x\|^{G}=\sup _{k}\left|x_{k}\right|^{G}, x=\left(x_{1}, x_{2}, x_{3}, \ldots\right) \in \lambda(G), \lambda \in\left\{\ell_{\infty}, c, c_{0}\right\} .
$$

Here, $G \lim$ is the geometric-limit. For the convenience, we denote $\ell_{\infty}(G), c(G), c_{0}(G)$, respectively as $l_{\infty}^{G}, c^{G}, c_{0}^{G}$.

In 1981, Kizmaz [13] introduced the notion of difference sequence spaces using forward difference operator $\Delta$ and studied the classical difference sequence spaces $\ell_{\infty}(\Delta), c(\Delta), c_{0}(\Delta)$. Following C. Türkmen and F. Başar [19], we defined geometric sequence space in [4] as follows:

$$
\ell_{\infty}^{G}\left(\Delta_{G}\right)=\left\{x=\left(x_{k}\right) \in \omega(G): \Delta_{G} x \in \ell_{\infty}^{G}\right\}, \text { where } \Delta_{G} x=x_{k} \ominus x_{k+1} .
$$

where $\Delta_{G} x=\left(\Delta_{G} x_{k}\right)=\left(x_{k} \ominus x_{k+1}\right)$.

In [4], we introduced some theorems, definitions and basic results as follows:

Theorem 2.1. The space $\ell_{\infty}^{G}\left(\Delta_{G}\right)$ is a normed linear space w.r.t. the norm

$$
\|x\|_{\Delta_{G}}^{G}=\left|x_{1}\right|^{G} \oplus\left\|\Delta_{G} x\right\|_{\infty}^{G} .
$$


Theorem 2.2. The space $\ell_{\infty}^{G}\left(\Delta_{G}\right)$ is a Banach space w.r.t. the norm $\|\cdot\|_{\Delta_{G}}^{G}$.

Remark 2.2. The spaces

(a) $c^{G}\left(\Delta_{G}\right)=\left\{\left(x_{k}\right) \in w(G): \Delta_{G} x_{k} \in c^{G}\right\}$

(b) $c_{0}^{G}\left(\Delta_{G}\right)=\left\{\left(x_{k}\right) \in w(G): \Delta_{G} x_{k} \in c_{0}^{G}\right\}$

are Banach spaces with respect to the norm $\|\cdot\|_{\Delta_{G}}^{G}$. Also these spaces are BK-spaces.

Lemma 2.3. The following conditions (a) and (b) are equivalent:

(a) $\sup _{k}\left|x_{k} \ominus x_{k+1}\right|^{G}<\infty$ i.e. $\sup _{k}\left|\Delta_{G} x_{k}\right|^{G}<\infty$;

(b) (i) $\sup _{k} e^{k^{-1}} \odot\left|x_{k}\right|^{G}<\infty$ and

(ii) $\sup _{k}\left|x_{k} \ominus e^{k(k+1)^{-1}} \odot x_{k+1}\right|^{G}<\infty$.

\section{Lemma 2.4.}

$$
\text { If } \sup _{n}\left|G \sum_{v=1}^{n} c_{v}\right|^{G} \leq \infty \text { then } \sup _{n}\left(p_{n} \odot\left|G \sum_{k=1}^{\infty} \frac{c_{n+k-1}}{p_{n+k}} G\right|^{G}\right)<\infty \text {. }
$$

Corollary 2.5. Let $\left(p_{n}\right)$ be monotonically increasing. If

$$
\sup _{n}\left|G \sum_{v=1}^{n} p_{v} \odot a_{v}\right|^{G}<\infty \text { then } \sup _{n}\left|p_{n} \odot{ }_{G} \sum_{k=n+1}^{\infty} a_{k}\right|^{G}<\infty .
$$

\section{Corollary 2.6.}

$$
\text { If } \sum_{k=1}^{\infty} p_{k} \odot a_{k} \text { is convergent then } \lim _{n} p_{n} \odot{ }_{G} \sum_{k=n+1}^{\infty} a_{k}=1 \text {. }
$$

Corollary 2.7. $G \sum_{k=1}^{\infty} e^{k} \odot a_{k}$ is convergent iff $\sum_{G} \sum_{k=1}^{\infty} R_{k}$ is convergent withe $^{n} \odot R_{n}=O(e)$, where

$$
R_{n}={ }_{G} \sum_{k=n+1}^{\infty} a_{k}
$$




\section{Main Results}

Following Kizmaz [13], generalized sequence spaces $\ell_{\infty}\left(\Delta^{m}\right), c\left(\Delta^{m}\right)$ and $c_{0}\left(\Delta^{m}\right)$ were introduced by Et and Çolak [7]. Based on Et and Çolak [7], Çolak and Et [6]. In [4] we introduced the geometric difference sequence. Now we define the following geometric generalized difference sequence spaces

$$
\begin{aligned}
\ell_{\infty}^{G}\left(\Delta_{G}^{m}\right) & =\left\{x=\left(x_{k}\right): \Delta_{G}^{m} x \in \ell_{\infty}^{G}\right\}, \\
c^{G}\left(\Delta_{G}^{m}\right) & =\left\{x=\left(x_{k}\right): \Delta_{G}^{m} x \in c^{G}\right\}, \\
c_{0}^{G}\left(\Delta_{G}^{m}\right) & =\left\{x=\left(x_{k}\right): \Delta_{G}^{m} x \in c_{0}^{G}\right\} .
\end{aligned}
$$

where $m \in \mathbf{N}$ and

$$
\begin{aligned}
& \Delta_{G}^{0} x=\left(x_{k}\right) \\
& \Delta_{G} x=\left(\Delta_{G} x_{k}\right)=\left(x_{k} \ominus x_{k+1}\right) \\
& \Delta_{G}^{2} x=\left(\Delta_{G}^{2} x_{k}\right)=\left(\Delta_{G} x_{k} \ominus \Delta_{G} x_{k+1}\right) \\
& =\left(x_{k} \ominus x_{k+1} \ominus x_{k+1} \oplus x_{k+2}\right) \\
& =\left(x_{k} \ominus e^{2} \odot x_{k+1} \oplus x_{k+1}\right) \\
& \Delta_{G}^{3} x=\left(\Delta_{G}^{3} x_{k}\right)=\left(\Delta_{G}^{2} x_{k} \ominus \Delta_{G}^{2} x_{k+1}\right) \\
& =\left(x_{k} \ominus e^{3} \odot x_{k+1} \oplus e^{3} \odot x_{k+1} \ominus x_{k+3}\right) \\
& \Delta_{G}^{m} x=\left(\Delta_{G}^{m} x_{k}\right)=\left(\Delta_{G}^{m-1} x_{k} \ominus \Delta_{G}^{m-1} x_{k+1}\right) \\
& =\left(G \sum_{v=0}^{m}(\ominus e)^{v_{G}} \odot e^{\left(\begin{array}{c}
m \\
v
\end{array}\right)} \odot x_{k+v}\right) \text {, with }(\ominus e)^{0_{G}}=e .
\end{aligned}
$$

Then it can be easily proved that $\ell_{\infty}^{G}\left(\Delta_{G}^{m}\right), c_{\infty}^{G}\left(\Delta_{G}^{m}\right)$ and $c_{0}^{G}\left(\Delta_{G}^{m}\right)$ are normed linear spaces with norm

$$
\|x\|_{\Delta_{G}}^{G}={ }_{G} \sum_{i=1}^{m}\left|x_{i}\right|^{G} \oplus\left\|\Delta_{G}^{m} x\right\|_{\infty}^{G} .
$$

Note: Throughout this paper often we write $G \sum_{k}$ instead of $\sum_{k=1}^{\infty}$ and $\lim _{n}$ instead of $\lim _{n \rightarrow \infty}$.

Definition 3.1 (Geometric Associative Algebra). An associative algebra is a vector space $A \subset \mathbf{R}(G)$, equipped with a bilinear map(called multiplication)

$$
\begin{array}{cc}
\odot: A \times A & \rightarrow A \\
(a, b) & \rightarrow a \odot b
\end{array}
$$


which is associative, i.e.

$$
(a \odot b) \odot c=a \odot(b \odot c) \forall a, b, c \in A .
$$

An algebra is commutative if $a \odot b=b \odot a$ for all $a, b \in A$. An algebra is unital if there exists a unique $e \in A$ such that $e \odot a=a \odot e=a$ for all $a \in A$. A subalgebra of the algebra $A$ is a subspace $B$ that is closed under multiplication, i.e. $a \odot b \in A$ for all $a, b \in B$.

Definition 3.2 (Geometric Normed Algebra). A normed algebra is a normed space $A \subset \omega(G)$ that is also an associative algebra, such that the norm is submultiplicative: $\|a \odot b\|^{G} \leq\|a\|^{G} \odot\|b\|^{G}$ for all $a, b \in A$. A geometric algebra is a complete normed algebra, i.e., a normed algebra which is also a Banach space with respect to its norm.

It is to be noted that the submultiplicativity of the norm means that multiplication in normed algebras is jointly continuous, i.e. if $a_{n} G a$ and $b_{n} G b$ then $\left(a_{n}\right)$ is bounded and

$$
\begin{gathered}
\left\|a_{n} \odot b_{n} \ominus a \odot b\right\|^{G}=\left\|a_{n} \odot\left(b_{n} \ominus b\right) \oplus\left(a_{n} \ominus a\right) \odot b\right\|^{G} \\
\leq\left\|a_{n}\right\|^{G} \odot\left\|b_{n} \ominus b\right\|^{G} \oplus\left\|a_{n} \ominus a\right\|^{G} \odot\|b\|^{G} \\
\leq \sup \left\{\left\|a_{n}\right\|^{G}\right\} \odot\left\|b_{n} \ominus b\right\|^{G} \oplus\|b\|^{G} \odot\left\|a_{n} \ominus a\right\|^{G} G 1 \text { as } n \rightarrow \infty .
\end{gathered}
$$

Definition 3.3 (Geometric Sequence Algebra). A geometric sequence space $E(G)$ is said to be sequence algebra if $x \odot y \in E(G)$ for $x=\left(x_{k}\right), y=$ $\left(y_{k}\right) \in E(G)$. i.e. $E(G)$ is closed under the geometric multiplication $\odot$ defined by

$$
\begin{gathered}
\odot: E(G) \times E(G) \rightarrow E(G) \\
(x, y) \rightarrow x \odot y=\left(x_{k}\right) \odot\left(y_{k}\right)=\left(x_{k}^{\ln y_{k}}\right)
\end{gathered}
$$

for any two sequences $x=\left(x_{k}\right), y=\left(y_{k}\right) \in E$.

Since $\omega(G)$ is closed under geometric multiplication $\odot$, hence, $\omega(G)$ is a sequence algebra. Also sequence algebra $\omega(G)$ is unital as $\left\|e_{G}\right\|^{G}=e$, where $e_{G}=(e, e, e, \ldots) \in \omega(G)$.

Definition 3.4 (Continuous Dual Space). If $X$ is a normed space, a linear map $f: X \rightarrow \mathbf{R}(G)$ is called linear functional. $f$ is called continuous linear functional or bounded linear functional if $\|f\|^{G}<\infty$, where

$$
\|f\|^{G}=\sup \left\{|f(x)|^{G}:\|x\|^{G} \leq e \text { for all } x \in X\right\} .
$$

Let $X^{*}$ be the collection of all bounded linear functionals on $X$. If $f, g \in X^{*}$ and $\alpha \in \mathbf{R}(G)$, we define $(\alpha \odot f \oplus g)(x)=\alpha \odot f(x) \oplus g(x) ; X^{*}$ is called the continuous dual space of $X$. 
Theorem 3.1. The sequence spaces $\ell_{\infty}^{G}\left(\Delta_{G}^{m}\right), c^{G}\left(\Delta_{G}^{m}\right)$ and $c_{0}^{G}\left(\Delta_{G}^{m}\right)$ are Banach spaces with the norm

$$
\|x\|_{\Delta_{G}}^{G}={ }_{G} \sum_{i=1}^{m}\left|x_{i}\right|^{G} \oplus\left\|\Delta_{G}^{m} x\right\|_{\infty}^{G} .
$$

Proof. Let $\left(x_{n}\right)$ be a Cauchy sequence in $\ell_{\infty}^{G}\left(\Delta_{G}^{m}\right)$, where $x_{n}=\left(x_{i}^{(n)}\right)=$ $\left(x_{1}^{(n)}, x_{2}^{(n)}, x_{3}^{(n)}, \ldots\right)$ for $n \in \mathbf{N}$ and $x_{k}^{(n)}$ is the $k^{\text {th }}$ coordinate of $x_{n}$. Then

$$
\begin{gathered}
\left\|x_{n} \ominus x_{l}\right\|_{\Delta_{G}}^{G}={ }_{G} \sum_{i=1}^{m}\left|x_{i}^{(n)} \ominus x_{i}^{(l)}\right|^{G} \oplus\left\|\Delta_{G}^{m}\left(x_{n} \ominus x_{l}\right)\right\|_{\infty}^{G} \\
={ }_{G} \sum_{i=1}^{m}\left|x_{i}^{(n)} \ominus x_{i}^{(l)}\right|^{G} \oplus \sup _{k}\left|\Delta_{G}^{m}\left(x_{n} \ominus x_{l}\right)\right|^{G} \rightarrow 1 \text { as } l, n \rightarrow \infty .
\end{gathered}
$$

Hence we obtain

$$
\left|x_{k}^{(n)} \ominus x_{k}^{(l)}\right|^{G} \rightarrow 1
$$

as $n, l \rightarrow \infty$ and for each $k \in \mathbf{N}$. Therefore $\left(x_{k}^{(n)}\right)=\left(x_{k}^{(1)}, x_{k}^{(2)}, x_{k}^{(3)}, \ldots\right)$ is a Cauchy sequence in $\mathbf{R}(G)$. Since $\mathbf{R}(G)$ is complete, $\left(x_{k}^{(n)}\right)$ is convergent.

Suppose $\lim _{n} x_{k}^{(n)}=x_{k}$, for each $k \in \mathbf{N}$. Since $\left(x_{n}\right)$ is a Cauchy sequence, for each $\epsilon>1$, there exists $N=N(\epsilon)$ such that $\left\|x_{n} \ominus x_{l}\right\|_{\Delta_{G}}^{G}<\epsilon$ for all $n, l \geq N$. Hence from (3.1)

$$
G \sum_{i=1}^{m}\left|x_{i}^{(n)} \ominus x_{i}^{(l)}\right|^{G}<\epsilon \text { and }\left|G \sum_{v=0}^{m}(\ominus e)^{v_{G}} \odot e^{\left(\begin{array}{c}
m \\
v
\end{array}\right)} \odot\left(x_{k+v}^{(n)} \ominus x_{k+v}^{(l)}\right)\right|^{G}<\epsilon
$$

for all $k \in \mathbf{N}$ and $n, l \geq N$. So we have

$$
\lim _{l} G \sum_{i=1}^{m}\left|x_{i}^{(n)} \ominus x_{i}^{(l)}\right|^{G}=\sum_{G} \sum_{i=1}^{m}\left|x_{i}^{(n)} \ominus x_{i}\right|^{G}<\epsilon
$$

and $\lim _{l}\left|{ }_{G} \Delta_{G}^{m}\left(x_{k}^{(n)} \ominus x_{k}^{(l)}\right)\right|^{G}=\left|{ }_{G} \Delta_{G}^{m}\left(x_{k}^{(n)} \ominus x_{k}\right)\right|^{G}<\epsilon, \forall n \geq N$.

This implies $\left\|x_{n} \ominus x\right\|_{\Delta_{G}}^{G}<\epsilon^{2}, \forall n \geq N$, that is $x_{n} \stackrel{G}{\rightarrow} x$ as $n \rightarrow \infty$, where $x=\left(x_{k}\right)$. Now we have to show that $x \in \ell_{\infty}^{G}\left(\Delta_{G}^{m}\right)$. We have 


$$
\begin{aligned}
& \left|\Delta_{G}^{m} x_{k}\right|^{G}=\left|G \sum_{v=0}^{m}(\ominus e)^{v_{G}} \odot e^{\left(\begin{array}{c}
m \\
v
\end{array}\right)} \odot x_{k+v}\right|^{G} \\
& =\left|G \sum_{v=0}^{m}(\ominus e)^{v_{G}} \odot e^{\left(\begin{array}{c}
m \\
v
\end{array}\right)} \odot\left(x_{k+v} \ominus x_{k+v}^{N} \oplus x_{k+v}^{N}\right)\right|^{G} \\
& \leq\left|G \sum_{v=0}^{m}(\ominus e)^{v_{G}} \odot e^{\left(\begin{array}{c}
m \\
v
\end{array}\right)} \odot\left(x_{k+v}^{N} \ominus x_{k+v}\right)\right|^{G} \oplus\left|G \sum_{v=0}^{m}(\ominus e)^{v_{G}} \odot e^{\left(\begin{array}{c}
m \\
v
\end{array}\right)} \odot x_{k+v}^{N}\right|^{G} \\
& \leq\left\|x^{N} \ominus x\right\|_{\Delta_{G}}^{G} \oplus\left|\Delta_{G}^{m} x_{k}^{N}\right|^{G}=\mathrm{O}(e) .
\end{aligned}
$$

Therefore we obtain $x \in \ell_{\infty}^{G}\left(\Delta_{G}^{m}\right)$. Hence $\ell_{\infty}^{G}\left(\Delta_{G}^{m}\right)$ is a Banach space.

It can be shown that $c^{G}\left(\Delta_{G}^{m}\right)$ and $c_{0}^{G}\left(\Delta_{G}^{m}\right)$ are closed subspaces of $\ell_{\infty}^{G}\left(\Delta_{G}^{m}\right)$. Therefore these sequence spaces are Banach spaces with the same norm defined for $\ell_{\infty}^{G}\left(\Delta_{G}^{m}\right)$, above.

Now we give some inclusion relations between these sequence spaces.

\section{Lemma 3.2.}

$$
\begin{array}{cc}
\text { (i) } & c_{0}^{G}\left(\Delta_{G}^{m}\right) c_{0}^{G}\left(\Delta_{G}^{m+1}\right) ; \\
(i i) & c^{G}\left(\Delta_{G}^{m}\right) c^{G}\left(\Delta_{G}^{m+1}\right) \\
(\text { iii }) & \ell_{\infty}^{G}\left(\Delta_{G}^{m}\right) \ell_{\infty}^{G}\left(\Delta_{G}^{m+1}\right)
\end{array}
$$

Proof. $\quad(i)$ Let $x \in c_{0}^{G}\left(\Delta_{G}^{m}\right)$. Since

$$
\begin{gathered}
\left|\Delta_{G}^{m+1} x_{k}\right|^{G}=\left|\Delta_{G}^{m} x_{k} \ominus \Delta_{G}^{m} x_{k+1}\right|^{G} \\
\leq\left|\Delta_{G}^{m} x_{k}\right|^{G} \oplus\left|\Delta_{G}^{m} x_{k+1}\right|^{G} \rightarrow 1 \text { as } k \rightarrow \infty .
\end{gathered}
$$

therefore we obtain $x \in c_{0}^{G}\left(\Delta_{G}^{m+1}\right)$. Thus $c_{0}^{G}\left(\Delta_{G}^{m}\right) \subset c_{0}^{G}\left(\Delta_{G}^{m+1}\right)$.

This inclusion is strict. For let

$$
x=\left(e^{k^{m}}\right)=\left(e, e^{2^{m}}, e^{3^{m}}, e^{4^{m}}, \ldots, e^{k^{m}}, \ldots\right) .
$$

Then $x \in c_{0}^{G}\left(\Delta_{G}^{m+1}\right)$ as $(m+1)^{\text {th }}$ geometric difference of $e^{k^{m}}$ is 1 (geometric zero). But $x \notin c_{0}^{G}\left(\Delta_{G}^{m}\right)$ as $m^{\text {th }}$ geometric difference of $e^{k^{m}}$ is a constant. Hence the inclusion is strict.

The proofs of $(i i)$ and (iii) are similar to that of $(i)$.

Lemma 3.3. (i) $c_{0}^{G}\left(\Delta_{G}^{m}\right) c^{G}\left(\Delta_{G}^{m}\right)$; 
(ii) $c^{G}\left(\Delta_{G}^{m}\right) \ell_{\infty}^{G}\left(\Delta_{G}^{m}\right)$

Proofs are similar to that of Lemma 3.2.

Furthermore, since the sequence spaces $\ell_{\infty}^{G}\left(\Delta_{G}^{m}\right), c^{G}\left(\Delta_{G}^{m}\right)$ and $c_{0}^{G}\left(\Delta_{G}^{m}\right)$ are Banach spaces with continuous coordinates, that is, $\left\|x_{n} \ominus x\right\|_{\Delta_{G}}^{G} \rightarrow 1$ implies $\left|x_{k}^{(n)} \ominus x_{k}\right|^{G} \rightarrow 1, \forall k \in \mathbf{N}$ as $n \rightarrow \infty$, these are also BK-spaces.

Remark 3.1. It can be easily proved that $c_{0}^{G}$ is a sequence algebra. But in general, $\ell_{\infty}^{G}\left(\Delta_{G}^{m}\right), c^{G}\left(\Delta_{G}^{m}\right)$ and $c_{0}^{G}\left(\Delta_{G}^{m}\right)$ are not sequence algebra. For let $x=\left(e^{k}\right), y=\left(e^{k^{m-1}}\right)$. Clearly $x, y \in c_{0}^{G}\left(\Delta_{G}^{m}\right)$. But

$$
x \odot y=\left(e^{k} \odot e^{k^{m-1}}\right)=\left(e^{k^{m}}\right) \notin c_{0}^{G}\left(\Delta_{G}^{m}\right) \text { for } m \geq 2,
$$

since $m^{\text {th }}$ geometric difference of $e^{k^{m}}$ is constant.

Let us define the operator

$$
D: \ell_{\infty}^{G}\left(\Delta_{G}^{m}\right) \rightarrow \ell_{\infty}^{G}\left(\Delta_{G}^{m}\right) \text { as }
$$

$D x=\left(1,1,1, \ldots, 1, x_{m+1}, x_{m+2}, \ldots\right)$, where

$x=\left(x_{1}, x_{2}, x_{3}, \ldots, x_{m}, x_{m+1}, \ldots\right) \in \ell_{\infty}^{G}\left(\Delta_{G}^{m}\right)$. It is trivial that $D$ is a bounded linear operator on $\ell_{\infty}^{G}\left(\Delta_{G}^{m}\right)$. Furthermore, the set

$D\left[\ell_{\infty}^{G}\left(\Delta_{G}^{m}\right)\right]=D \ell_{\infty}^{G}\left(\Delta_{G}^{m}\right)=\left\{x=\left(x_{k}\right): x \in \ell_{\infty}^{G}\left(\Delta_{G}^{m}\right), x_{1}=x_{2}=\ldots=x_{m}=1\right\}$

is a subspace of $\ell_{\infty}^{G}\left(\Delta_{G}^{m}\right)$ and

$$
\begin{aligned}
\|x\|_{\Delta_{G}}^{G} & =\left|x_{1}\right|^{G} \oplus\left|x_{2}\right|^{G} \oplus \ldots \oplus\left|x_{m}\right|^{G} \oplus\left\|\Delta_{G}^{m} x\right\|_{\infty}^{G} \\
& =1 \oplus 1 \oplus \ldots \oplus 1 \oplus\left\|\Delta_{G}^{m} x\right\|_{\infty}^{G} \\
& =\left\|\Delta_{G}^{m} x\right\|_{\infty}^{G} \text { in } D \ell_{\infty}^{G}\left(\Delta_{G}^{m}\right) .
\end{aligned}
$$

Now let us define

$$
\begin{gathered}
\Delta^{m}: D \ell_{\infty}^{G}\left(\Delta_{G}^{m}\right) \rightarrow \ell_{\infty}^{G} \\
\Delta_{G}^{m} x=y=\left(\Delta_{G}^{m-1} x_{k} \ominus \Delta_{G}^{m-1} x_{k+1}\right) .
\end{gathered}
$$


$\Delta_{G}^{m}$ is a linear homomorphism: Let $x, y \in D \ell_{\infty}^{G}\left(\Delta_{G}^{m}\right)$. Then

$$
\begin{aligned}
& \Delta_{G}^{m}\left(x_{k} \oplus y_{k}\right)={ }_{G} \sum_{v=0}^{m}(\ominus e)^{v_{G}} \odot e^{\left(\begin{array}{c}
m \\
v
\end{array}\right)} \odot\left(x_{k} \oplus y_{k}\right) \\
& ={ }_{G} \sum_{v=0}^{m}(\ominus e)^{v_{G}} \odot e^{\left(\begin{array}{c}
m \\
v
\end{array}\right)} \odot x_{k} \oplus_{G} \sum_{v=0}^{m}(\ominus e)^{v_{G}} \odot e^{\left(\begin{array}{c}
m \\
v
\end{array}\right)} \odot y_{k} \\
& =\Delta_{G}^{m} x_{k} \oplus \Delta_{G}^{m} y_{k} \\
& \Delta_{G}^{m}(x \oplus y)=\Delta_{G}^{m} x \oplus \Delta_{G}^{m} y \text {. For } \alpha \in \mathbf{R}(G) \\
& \Delta_{G}^{m}(\alpha \odot x)=\left(\Delta_{G}^{m} \alpha \odot x_{k}\right) \\
& =\left(G \sum_{v=0}^{m}(\ominus e)^{v_{G}} \odot e^{\left(\begin{array}{c}
m \\
v
\end{array}\right)} \odot \alpha \odot x_{k}\right) \\
& =\left(\alpha \odot_{G} \sum_{v=0}^{m}(\ominus e)^{v_{G}} \odot e^{\left.\left(\begin{array}{c}
m \\
v
\end{array}\right) \odot x_{k}\right)}\right. \\
& =\alpha \odot \Delta_{G}^{m} \odot x \text {. }
\end{aligned}
$$

This implies that $\Delta_{G}^{m}$ is a linear homomorphism. Hence $D \ell_{\infty}^{G}\left(\Delta_{G}^{m}\right)$ and $\ell_{\infty}^{G}$ are equivalent as topological spaces [16]. $\Delta_{G}^{m}$ and $\left(\Delta_{G}^{m}\right)^{-1}$ are norm preserving and

$$
\left\|\Delta_{G}^{m}\right\|_{\infty}^{G}=\left\|\left(\Delta_{G}^{m}\right)^{-1}\right\|_{\infty}^{G}=e .
$$

Let $\left[\ell_{\infty}^{G}\right]^{\prime}$ and $\left[D \ell_{\infty}^{G}\left(\Delta_{G}^{m}\right)\right]^{\prime}$ denote the continuous duals of $\ell_{\infty}^{G}$ and $D \ell_{\infty}^{G}\left(\Delta_{G}^{m}\right)$, respectively.

It can be shown that

$$
\begin{aligned}
& s:\left[D \ell_{\infty}^{G}\left(\Delta_{G}^{m}\right)\right]^{\prime} \rightarrow\left[\ell_{\infty}^{G}\right]^{\prime} \\
& f_{\Delta} \rightarrow f_{\Delta} \circ\left(\Delta_{G}^{m}\right)^{-1}=f
\end{aligned}
$$

is a linear isometry. So $\left[D \ell_{\infty}^{G}\left(\Delta_{G}^{m}\right)\right]^{\prime}$ is equivalent to $\left[\ell_{\infty}^{G}\right]^{\prime}$.

In the same way, it can be shown that $D c^{G}\left(\Delta_{G}^{m}\right)$ and $D c_{0}^{G}\left(\Delta_{G}^{m}\right)$ are equivalent as topological space to $c^{G}$ and $c_{0}^{G}$, respectively. Also

$$
\left[D c^{G}\left(\Delta_{G}^{m}\right)\right]^{\prime} \cong\left[D c_{0}^{G}\left(\Delta_{G}^{m}\right)\right]^{\prime} \cong \ell_{1}^{G}
$$

where $\ell_{1}^{G}=\left\{x=\left(x_{k}\right):_{G} \sum_{k}\left|x_{k}\right|^{G}<\infty\right\}$.

Lemma 3.4. The following conditions (a) and (b) are equivalent:

(a) $\sup _{k}\left|x_{k} \ominus x_{k+1}\right|^{G}<\infty$ i.e. $\sup _{k}\left|\Delta_{G} x_{k}\right|^{G}<\infty$;

(b)

(i) $\sup _{k} e^{k^{-1}} \odot\left|x_{k}\right|^{G}<\infty$ and

(ii) $\sup _{k}\left|x_{k} \ominus e^{k(k+1)^{-1}} \odot x_{k+1}\right|^{G}<\infty$. 
Proof. Let (a) be true i.e. $\sup _{k}\left|x_{k} \ominus x_{k+1}\right|^{G}<\infty$.

Now $\left|x_{1} \ominus x_{k+1}\right|^{G}=\left|{ }_{G} \sum_{v=1}^{k}\left(x_{v} \ominus x_{v+1}\right)\right|^{G}$

$$
\begin{aligned}
& =\left|G \sum_{v=1}^{k} \Delta_{G} x_{v}\right|^{G} \\
& \leq G \sum_{v=1}^{k}\left|\Delta_{G} x_{v}\right|^{G}=O\left(e^{k}\right) \\
\text { and }\left|x_{k}\right|^{G}= & \left|x_{1} \ominus x_{1} \oplus x_{k+1} \oplus x_{k} \ominus x_{k+1}\right|^{G} \\
& \leq\left|x_{1}\right|^{G} \oplus\left|x_{1} \ominus x_{k+1}\right|^{G} \oplus\left|x_{k} \ominus x_{k+1}\right|^{G}=O\left(e^{k}\right) .
\end{aligned}
$$
$b(i)$.

This implies that $\sup _{k} e^{k^{-1}} \odot\left|x_{k}\right|^{G}<\infty$. This completes the proof of

\section{Again}

$$
\begin{aligned}
& \sup _{k}\left|x_{k} \ominus e^{k(k+1)^{-1}} \odot x_{k+1}\right|^{G}=\left|\left\{e^{(k+1)} \odot e^{(k+1)^{-1}}\right\} \odot x_{k} \ominus e^{k(k+1)^{-1}} \odot x_{k+1}\right|^{G} \\
&=\left|\left\{\left(e^{k} \oplus e\right) \odot e^{(k+1)^{-1}}\right\} \odot x_{k} \ominus e^{k(k+1)^{-1}} \odot x_{k+1}\right|^{G} \\
&=\left|\left\{e^{k(k+1)^{-1}} \odot x_{k} \oplus e^{(k+1)^{-1}} \odot x_{k}\right\} \ominus e^{k(k+1)^{-1}} \odot x_{k+1}\right|^{G} \\
&=\left|\left\{e^{k(k+1)^{-1}} \odot\left(x_{k} \ominus x_{k+1}\right)\right\} \oplus\left\{e^{(k+1)^{-1}} \odot x_{k}\right\}\right|^{G} \\
& \leq e^{k(k+1)^{-1}} \odot\left|x_{k} \ominus x_{k+1}\right|^{G} \oplus e^{(k+1)^{-1}} \odot\left|x_{k}\right|^{G} \\
&=O(e) .
\end{aligned}
$$

Therefore $\sup _{k}\left|x_{k} \ominus e^{k(k+1)^{-1}} \odot x_{k+1}\right|^{G}<\infty$. This completes the proof of $b(i i)$.

Conversely let $(b)$ be true. Then

$$
\begin{aligned}
& \left|x_{k} \ominus e^{k(k+1)^{-1}} \odot x_{k+1}\right|^{G}=\left|e^{(k+1)(k+1)^{-1}} \odot x_{k} \ominus e^{k(k+1)^{-1}} \odot x_{k+1}\right|^{G} \\
& \geq e^{k(k+1)^{-1}} \odot\left|x_{k} \ominus x_{k+1}\right|^{G} \ominus e^{(k+1)^{-1}} \odot\left|x_{k}\right|^{G}
\end{aligned}
$$

Thus $\sup _{k}\left|x_{k} \ominus x_{k+1}\right|^{G}<\infty$ as $b(i)$ and $b(i i)$ hold.

Corollary 3.5. The following conditions $(a)$ and $(b)$ are equivalent

(a) $\sup _{k}\left|\Delta_{G}^{m-1} x_{k} \ominus \Delta_{G}^{m-1} x_{k+1}\right|^{G}<\infty$;

(b) $\quad(i) \sup _{k} e^{k^{-1}} \odot\left|\Delta_{G}^{m-1} x_{k}\right|^{G}<\infty$

$$
\text { (ii) } \sup _{k}\left|\Delta_{G}^{m-1} x_{k} \ominus e^{k(k+1)^{-1}} \odot \Delta_{G}^{m-1} x_{k+1}\right|^{G}<\infty .
$$


Proof. By putting $\Delta_{G}^{m-1} x_{k}$ instead of $x_{k}$ in Lemma 3.4, results are obvious.

\section{Lemma 3.6.}

$$
\sup _{k} e^{k^{-i}} \odot\left|\Delta_{G} x_{k}\right|^{G}<\infty \text { implies } \sup _{k} e^{-(i+1)} \odot\left|x_{k}\right|^{G}<\infty, \forall i \in \mathbf{N} .
$$

Proof. For $i=1$ it is obvious from the Lemma 3.4. Let the result be true for $i=n$. i.e. $\sup _{k} e^{k^{-n}} \odot\left|\Delta_{G} x_{k}\right|^{G}<\infty$. Then

$$
\begin{aligned}
& \left|x_{k} \ominus x_{k+1}\right|^{G}=\left|G \sum_{v=1}^{k} \Delta_{G} x_{k}\right|^{G} \\
& \leq_{G} \sum_{v=1}^{k}\left|\Delta_{G} x_{k}\right|^{G}=\mathrm{O}\left(\left(e^{k^{n}}\right)^{k}\right)=\mathrm{O}\left(e^{k^{(n+1)}}\right), \\
& \text { as } \sup _{k} e^{k^{-n}} \odot\left|\Delta_{G} x_{k}\right|^{G}<\infty \\
& \text { and }-\left.\mathrm{x}_{k}\right|^{G}=\left|x_{k} \oplus x_{1} \ominus x_{1} \oplus x_{k+1} \ominus x_{k+1}\right|^{G} \\
& \leq\left|x_{1}\right|^{G} \oplus\left|x_{1} \ominus x_{k+1}\right|^{G} \oplus\left|x_{k} \ominus x_{k+1}\right|^{G}=\mathrm{O}\left(e^{k^{(n+1)}}\right) .
\end{aligned}
$$

From this we obtain, $\sup _{k} e^{k^{-(n+1)}} \odot\left|x_{k}\right|^{G}<\infty$. Thus $\sup _{k} e^{k^{-(i+1)}} \odot\left|x_{k}\right|^{G}<$ $\infty, \forall i \in \mathbf{N}$.

\section{Lemma 3.7.}

$\sup _{k} e^{k^{-i}} \odot\left|\Delta_{G}^{m-1} x_{k}\right|^{G}<\infty$ implies

$\sup _{k} e^{-(i+1)} \odot\left|\Delta_{G}^{m-(i+1)} x_{k}\right|^{G}<\infty, \forall i, m \in \mathbf{N}$ and $1 \leq i<m$.

Proof. Putting $\Delta_{G}^{m-i} x_{k}$ instead of $\Delta_{G} x_{k}$ in Lemma 3.6, the result is immediate.

Corollary 3.8. $\sup _{k} e^{k^{-1}} \odot\left|\Delta_{G}^{m-1} x_{k}\right|<\infty$ implies $\sup _{k} e^{k^{-m}} \odot\left|x_{k}\right|<\infty$.

Proof. In Lemma 3.7, putting $i=1$, we get

Similarly,

$$
\sup _{k} e^{k^{-1}} \odot\left|\Delta_{G}^{m-1} x_{k}\right|^{G}<\infty \Rightarrow \sup _{k} e^{k^{-2}} \odot\left|\Delta_{G}^{m-2} x_{k}\right|^{G}<\infty
$$

$$
\sup _{k} e^{k^{-2}} \odot\left|\Delta_{G}^{m-2} x_{k}\right|^{G}<\infty \Rightarrow \sup _{k} e^{k^{-3}} \odot\left|\Delta_{G}^{m-3} x_{k}\right|^{G}<\infty .
$$

Continuing the process we get

Thus

$$
\sup _{k} e^{k^{-(m-1)}} \odot\left|\Delta_{G}^{1} x_{k}\right|^{G}<\infty \Rightarrow \sup _{k} e^{k^{-m}} \odot\left|\Delta_{G}^{0} x_{k}\right|^{G}<\infty
$$

$$
\sup _{k} e^{k^{-1}} \odot\left|\Delta_{G}^{m-1} x_{k}\right|^{G}<\infty \Rightarrow \sup _{k} e^{k^{-m}} \odot\left|x_{k}\right|^{G}<\infty .
$$

Corollary 3.9. If $x \in \ell_{\infty}^{G}\left(\Delta_{G}^{m}\right)$ then $\sup _{k} e^{k^{-m}} \odot\left|x_{k}\right|^{G}<\infty$. 


\section{Proof.}

$$
\begin{aligned}
x \in \ell_{\infty}^{G}\left(\Delta_{G}^{m}\right) & \Rightarrow \Delta_{G}^{m} x \in \ell_{\infty}^{G} \\
& \Rightarrow \sup _{k}\left|\Delta_{G}^{m} x_{k}\right|^{G}<\infty \\
& \Rightarrow \sup _{k}\left|\Delta_{G}^{m-1} x_{k} \ominus \Delta_{G}^{m-1} x_{k+1}\right|^{G}<\infty \\
& \Rightarrow \sup _{k} e^{k^{-1}} \odot\left|\Delta_{G}^{m} x_{k}\right|^{G}<\infty \quad \text { by Corollary } 3.5 \\
& \Rightarrow \sup _{k} e^{k^{-m}} \odot\left|x_{k}\right|^{G}<\infty \text { by Corollary } 3.8 .
\end{aligned}
$$

\section{4. $\alpha-, \beta-, \gamma-$ duals}

Definition 4.1. $[8,14,15,16]$ If $X$ is a sequence space, it is defined that

(i) $X^{\alpha}=\left\{a=\left(a_{k}\right): \sum_{k=1}^{\infty}\left|a_{k} x_{k}\right|<\infty\right.$, for each $\left.x \in X\right\}$;

(ii) $X^{\beta}=\left\{a=\left(a_{k}\right): \sum_{k=1}^{\infty} a_{k} x_{k}\right.$ is convergent, for each $\left.x \in X\right\}$;

(iii) $X^{\gamma}=\left\{a=\left(a_{k}\right): \sup _{n}\left|\sum_{k=1}^{n} a_{k} x_{k}\right|<\infty\right.$, for each $\left.x \in X\right\}$.

Then $X^{\alpha}, X^{\beta}$ and $X^{\gamma}$ are called $\alpha$-dual (or Köthe-Toeplitz dual), $\beta$-dual (or generalized Köthe-Toeplitz dual) and $\gamma$-dual spaces of $X$, re-

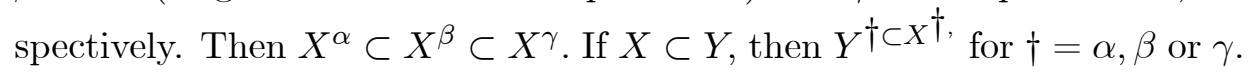
It is clear that $X \subset\left(X^{\alpha}\right)^{\alpha}=X^{\alpha \alpha}$. If $X=X^{\alpha \alpha}$ then $X$ is called $\alpha$-space. $\alpha$-space is also called a Köthe space or a perfect sequence space.

Then we defined and have proved that [4]

$\left(s \ell_{\infty}^{G}\left(\Delta_{G}\right)\right)^{\alpha}=\left\{a=\left(a_{k}\right):_{G} \sum_{k=1}^{\infty} e^{k} \odot\left|a_{k}\right|^{G}<\infty\right\}$

$\left(s \ell_{\infty}^{G}\left(\Delta_{G}\right)\right)^{\beta}=\left\{a=\left(a_{k}\right): G \sum_{k=1}^{\infty} e^{k} \odot a_{k}\right.$ is convergent with $\left.G \sum_{k=1}^{\infty}\left|R_{k}\right|^{G}<\infty\right\}$

$\left(s \ell_{\infty}^{G}\left(\Delta_{G}\right)\right)^{\gamma}=\left\{a=\left(a_{k}\right): \sup _{n}\left|G \sum_{k=1}^{n} e^{k} \odot a_{k}\right|^{G}<\infty, G \sum_{k=1}^{\infty}\left|R_{k}\right|^{G}<\infty\right\}$,

where $R_{k}=G \sum_{n=k+1}^{\infty} a_{n}$ and $s: \ell_{\infty}^{G}\left(\Delta_{G}\right) \rightarrow \ell_{\infty}^{G}\left(\Delta_{G}\right), x \rightarrow s x=y=$ $\left(1, x_{2}, x_{3}, \ldots\right)$.

Theorem 4.1. [4]

(i) If $D_{1}=\left\{a=\left(a_{k}\right): G \sum_{k=1}^{\infty} e^{k} \odot\left|a_{k}\right|^{G}<\infty\right\}$ then $\left(s \ell_{\infty}^{G}\left(\Delta_{G}\right)\right)^{\alpha}=D_{1}$. 
(ii) If $D_{2}=\left\{a=\left(a_{k}\right):{ }_{G} \sum_{k=1}^{\infty} e^{k} \odot a_{k}\right.$ is convergent with $\left.G \sum_{k=1}^{\infty}\left|R_{k}\right|^{G}<\infty\right\}$.

Then $\left(s \ell_{\infty}^{G}\left(\Delta_{G}\right)\right)^{\beta}=D_{2}$.

(iii) If $D_{3}=\left\{a=\left(a_{k}\right): \sup _{n}\left|G \sum_{k=1}^{n} e^{k} \odot a_{k}\right|^{G}<\infty, G \sum_{k=1}^{\infty}\left|R_{k}\right|^{G}<\infty\right\}$.

Then $\left(s \ell_{\infty}^{G}\left(\Delta_{G}\right)\right)^{\gamma}=D_{3}$.

Lemma 4.2. Let $U_{1}=\left\{a=\left(a_{k}\right):{ }_{G} \sum_{k} e^{k^{m}} \odot\left|a_{k}\right|^{G}<\infty\right\}$. Then $\left[D \ell_{\infty}^{G}\left(\Delta_{G}^{m}\right)\right]^{\alpha}=U_{1}$.

Proof. Let $a \in U_{1}$, then using Corollary 3.5, for $x \in D \ell_{\infty}^{G}\left(\Delta_{G}^{m}\right)$, we have $G \sum_{k}\left|a_{k} \odot x_{k}\right|^{G}={ }_{G} \sum_{k}\left\{e^{k^{m}} \odot\left|a_{k}\right|^{G}\right\} \odot\left\{e^{k^{-m}} \odot\left|x_{k}\right|^{G}\right\}<\infty$ by Corollary (3.8).

This implies that $a \in\left[D \ell_{\infty}^{G}\left(\Delta_{G}^{m}\right)\right]^{\alpha}$. Therefore

$$
U_{1} \subseteq\left[D \ell_{\infty}^{G}\left(\Delta_{G}^{m}\right)\right]^{\alpha}
$$

Conversely, let $a \in\left[D \ell_{\infty}^{G}\left(\Delta_{G}^{m}\right)\right]^{\alpha}$. Then ${ }_{G} \sum_{k}\left|a_{k} \odot x_{k}\right|^{G}<\infty$ (by definition of $\alpha$-dual) for $x \in D \ell_{\infty}^{G}\left(\Delta_{G}^{m}\right)$. So we take

$$
x_{k}=\begin{array}{cl}
1, & \text { if } k \leq m \\
e^{k^{m}}, & \text { if } k>m
\end{array}
$$

Then $x=\left(1,1,1, \ldots, 1, e^{(m+1)^{m}}, e^{(m+2)^{m}}, \ldots\right) \in D \ell_{\infty}^{G}\left(\Delta_{G}^{m}\right)$. Therefore

$$
\begin{gathered}
G \sum_{k=1}^{\infty} e^{k^{m}} \odot\left|a_{k}\right|^{G}={ }_{G} \sum_{k=1}^{m} e^{k^{m}} \odot\left|a_{k}\right|^{G} \oplus G \sum_{k=m+1}^{\infty} e^{k^{m}} \odot\left|a_{k}\right|^{G} \\
=G \sum_{k=1}^{m} e^{k^{m}} \odot\left|a_{k}\right|^{G} \oplus G \sum_{k=1}^{\infty}\left|a_{k} \odot x_{k}\right|^{G}<\infty
\end{gathered}
$$

since $a_{k} \odot x_{k}=1$ (the geometric zero) for $k=1,2, \ldots, m$.

Therefore $a \in U_{1}$. This implies

$$
\left[D \ell_{\infty}^{G}\left(\Delta_{G}^{m}\right)\right]^{\alpha} \subseteq U_{1}
$$

Then from (4.1) and (4.3), we get $\left[D \ell_{\infty}^{G}\left(\Delta_{G}^{m}\right)\right]^{\alpha}=U_{1}$.

\section{Lemma 4.3.}

$$
\left[D \ell_{\infty}^{G}\left(\Delta_{G}^{m}\right)\right]^{\alpha}=\left[D c^{G}\left(\Delta_{G}^{m}\right)\right]^{\alpha}
$$


Proof. Since $D c^{G}\left(\Delta_{G}^{m}\right) \subseteq D \ell_{\infty}^{G}\left(\Delta_{G}^{m}\right)$, hence $\left[D \ell_{\infty}^{G}\left(\Delta_{G}^{m}\right)\right]^{\alpha} \subseteq\left[D c^{G}\left(\Delta_{G}^{m}\right)\right]^{\alpha}$.

Again let $a \in\left[D c^{G}\left(\Delta_{G}^{m}\right)\right]^{\alpha}$. Then $G \sum_{k}\left|a_{k} \odot x_{k}\right|^{G}<\infty$ for each $x \in$ $D c^{G}\left(\Delta_{G}^{m}\right)$. If we take $x=\left(x_{k}\right)$ which is defined in (4.2), we get

$$
G \sum_{k} e^{k^{m}} \odot\left|a_{k}\right|^{G}=G \sum_{k=1}^{m} e^{k^{m}} \odot\left|a_{k}\right|^{G} \oplus G \sum_{k}\left|a_{k} \odot x_{k}\right|^{G}<\infty .
$$

This implies that $a \in\left[D \ell_{\infty}^{G}\left(\Delta_{G}^{m}\right)\right]^{\alpha}$. Thus

$$
\left[D \ell_{\infty}^{G}\left(\Delta_{G}^{m}\right)\right]^{\alpha}=\left[D c^{G}\left(\Delta_{G}^{m}\right)\right]^{\alpha}
$$

\section{Lemma 4.4.}

(i) $\left[\ell_{\infty}^{G}\left(\Delta_{G}^{m}\right)\right]_{\alpha}^{\alpha}=\left[D \ell_{\infty}^{G}\left(\Delta_{G}^{m}\right)\right]_{\alpha}^{\alpha}$.

(ii) $\left[c^{G}\left(\Delta_{G}^{m}\right)\right]^{\alpha}=\left[D c^{G}\left(\Delta_{G}^{m}\right)\right]^{\alpha}$.

Proof. (i) Since $D \ell_{\infty}^{G}\left(\Delta_{G}^{m}\right) \subseteq \ell_{\infty}^{G}\left(\Delta_{G}^{m}\right)$, so $\left[\ell_{\infty}^{G}\left(\Delta_{G}^{m}\right)\right]^{\alpha} \subseteq\left[D \ell_{\infty}^{G}\left(\Delta_{G}^{m}\right)\right]^{\alpha}$. Let $a \in\left[D \ell_{\infty}^{G}\left(\Delta_{G}^{m}\right)\right]^{\alpha}$ and $x \in \ell_{\infty}^{G}\left(\Delta_{G}^{m}\right)$. From Corollary 3.9, we have

$$
G \sum_{k}\left|a_{k} \odot x_{k}\right|^{G}=G \sum_{k} e^{k^{m}} \odot\left|a_{k}\right|^{G} \odot\left(e^{k^{-m}} \odot\left|x_{k}\right|^{G}\right)<\infty .
$$

Hence $a \in\left[\ell_{\infty}^{G}\left(\Delta_{G}^{m}\right)\right]^{\alpha}$.

(ii) $D c^{G}\left(\Delta_{G}^{m}\right) \subseteq c^{G}\left(\Delta_{G}^{m}\right)$ implies $\left[c^{G}\left(\Delta_{G}^{m}\right)\right]^{\alpha} \subseteq\left[D c^{G}\left(\Delta_{G}^{m}\right)\right]^{\alpha}$.

Let $a \in\left[D c^{G}\left(\Delta_{G}^{m}\right)\right]^{\alpha}$ and $x \in c^{G}\left(\Delta_{G}^{m}\right)$. From Corollary 3.9, we have

$$
G \sum_{k}\left|a_{k} \odot x_{k}\right|^{G}={ }_{G} \sum_{k} e^{k^{m}} \odot\left|a_{k}\right|^{G} \odot\left(e^{k^{-m}} \odot\left|x_{k}\right|^{G}\right)<\infty
$$

for $x \in c^{G}\left(\Delta_{G}^{m}\right) \subseteq l_{\infty}^{G}\left(\Delta_{G}^{m}\right)$. Hence $a \in\left[c^{G}\left(\Delta_{G}^{m}\right)\right]^{\alpha}$. This completes the proof.

Theorem 4.5. Let $X$ stand for $\ell_{\infty}^{G}$ or $c^{G}$. Then

$$
\left[X\left(\Delta_{G}^{m}\right)\right]^{\alpha}=\left\{a=\left(a_{k}\right): G \sum_{k} e^{k^{m}} \odot\left|a_{k}\right|^{G}<\infty\right\} .
$$


Proof.

$$
\begin{aligned}
{\left[\ell_{\infty}^{G}\left(\Delta_{G}^{m}\right)\right]^{\alpha} } & =\left[D \ell_{\infty}^{G}\left(\Delta_{G}^{m}\right)\right]^{\alpha} \quad \text { by Lemma } 4.4 \\
& =\left\{a=\left(a_{k}\right): G \sum_{k} e^{k^{m}} \odot\left|a_{k}\right|^{G}<\infty\right\} \text { by Lemma 4.2. }
\end{aligned}
$$

Again

$$
\begin{aligned}
{\left[c^{G}\left(\Delta_{G}^{m}\right)\right]^{\alpha} } & =\left[D c^{G}\left(\Delta_{G}^{m}\right)\right]^{\alpha} \quad \text { by Lemma } 4.4 \\
& =\left[D \ell_{\infty}^{G}\left(\Delta_{G}^{m}\right)\right]^{\alpha} \quad \text { by Lemma } 4.3 \\
& =\left\{a=\left(a_{k}\right):{ }_{G} \sum_{k} e^{k^{m}} \odot\left|a_{k}\right|^{G}<\infty\right\} \text { by Lemma 4.2. }
\end{aligned}
$$

Corollary 4.6. For $X=\ell_{\infty}^{G}$ or $c^{G}$, then

$$
\begin{gathered}
{\left[X\left(\Delta_{G}\right)\right]^{\alpha}=\left\{a=\left(a_{k}\right): G \sum_{k} e^{k} \odot\left|a_{k}\right|^{G}<\infty\right\}, \text { and }} \\
{\left[X\left(\Delta_{G}^{2}\right)\right]^{\alpha}=\left\{a=\left(a_{k}\right): G \sum_{k} e^{k^{2}} \odot\left|a_{k}\right|^{G}<\infty\right\} .}
\end{gathered}
$$

Proof. Putting $m=1$ and $m=2$ in Theorem 4.5, the results follow.

Theorem 4.7. Let $X$ stand for $\ell_{\infty}^{G}$ or $c^{G}$ and $U_{2}=\left\{a=\left(a_{k}\right): \sup _{k} e^{k^{-m}} \odot\right.$ $\left.\left|a_{k}\right|^{G}<\infty\right\}$. Then $\left[X\left(\Delta_{G}^{m}\right)\right]^{\alpha \alpha}=U_{2}$.

Proof. Let $a \in U_{2}$ and $x \in\left[X\left(\Delta_{G}^{m}\right)\right]^{\alpha}$, then by definition of $U_{2}$ and by Lemma 4.2 , we get

$$
\begin{aligned}
& G \sum_{k}\left|a_{k} \odot x_{k}\right|^{G}=_{G} \sum_{k} e^{k^{m}} \odot\left|x_{k}\right|^{G} \odot e^{k^{-m}} \odot\left|a_{k}\right|^{G} \\
& \leq_{G} \sum_{k} e^{k^{m}} \odot\left|x_{k}\right|^{G} \odot \sup _{k} e^{k^{-m}} \odot\left|a_{k}\right|^{G}<\infty .
\end{aligned}
$$

Hence $a \in\left[X\left(\Delta_{G}^{m}\right)\right]^{\alpha \alpha}$.

Conversely, let $a \in\left[X\left(\Delta_{G}^{m}\right)\right]^{\alpha \alpha}$ and $a \notin U_{2}$. Then we must have

$$
\sup _{k} e^{k^{-m}} \odot\left|a_{k}\right|^{G}=\infty .
$$

Hence there exists a strictly increasing sequence $\left(e^{k(i)}\right)$ of geometric integers (see [19]), where $k(i)$ is a strictly increasing sequence of positive integers such that

$$
e^{[k(i)]^{-m}} \odot\left|a_{k(i)}\right|^{G}>e^{i^{m}} .
$$


Let us define the sequence $x$ by

$$
\mathrm{x}_{k}=\left\{\begin{array}{cc}
\left(\left|a_{k(i)}\right|^{G}\right)^{-1_{G}}, & k=k(i) \\
1, & k \neq k(i) .
\end{array}\right.
$$

where $\left(\left|a_{k(i)}\right|^{G}\right)^{-1_{G}}$ is the geometric inverse of $\left|a_{k(i)}\right|^{G}$ so that $\left|a_{k(i)}\right|^{G} \odot$ $\left(\left|a_{k(i)}\right|^{G}\right)^{-1_{G}}=e$.

Then we have

$$
G \sum_{k} e^{k^{m}} \odot\left|x_{k}\right|^{G}=G \sum_{i} e^{[k(i)]^{m}} \odot\left[\left|a_{k(i)}\right|^{G}\right]^{-1_{G}} \leq e^{i^{-m}}<\infty .
$$

Hence $x \in\left[X\left(\Delta_{G}^{m}\right)\right]^{\alpha}$ and $\sum_{G} \sum_{k}\left|a_{k} \odot x_{k}\right|^{G}=\sum e=\infty$. This is a contradiction as $a \in\left[X\left(\Delta_{G}^{m}\right)\right]^{\alpha \alpha}$. Hence $a \in U_{2}$.

Corollary 4.8. For $X=\ell_{\infty}^{G}$ or $c^{G}$, then

$$
\left[X\left(\Delta_{G}^{2}\right)\right]^{\alpha \alpha}=\left\{a=\left(a_{k}\right): \sup _{k} e^{k^{-2}} \odot\left|a_{k}\right|^{G}<\infty\right\} .
$$

Proof. In Theorem (4.7), putting $m=2$ we obtain the result.

Corollary 4.9. The sequence spaces $\ell_{\infty}^{G}\left(\Delta_{G}^{m}\right)$ and $c^{G}\left(\Delta_{G}^{m}\right)$ are not perfect.

Proof. Proof is trivial as $X^{\alpha \alpha} \neq X$ for $X=\ell_{\infty}^{G}\left(\Delta_{G}^{m}\right)$ or $c^{G}\left(\Delta_{G}^{m}\right)$.

\section{Conclusion}

In this paper, we have defined geometric generalized difference sequence space and obtained some inclusion results between these spaces. We think that geometric calculus may especially be useful as a mathematical tool for economics, management and finance.

\section{References}

[1] A. E. Bashirov, E. Mısırlı, Y. Tandoğdu, A. Özyapıcı, On modeling with multiplicative differential equations, Appl. Math. J. Chinese Univ. 26 (4), pp. 425-438, (2011). 
[2] A. E. Bashirov, E. M. Kurpınar, A. Özyapici, Multiplicative Calculus and its applications, J. Math. Anal. Appl. 337, pp. 36-48, (2008).

[3] A. E. Bashirov, M. Riza, On Complex multiplicative differentiation, TWMS J. App. Eng. Math. 1 (1), pp. 75-85, (2011).

[4] K. Boruah, B. Hazarika, Application of Geometric Calculus in Numerical Analysis and Difference Sequence Spaces, J. Math. Anal. Appl 449 (2), pp. 1265-1285, (2017).

[5] A. F. Çakmak, F. Başar, On Classical sequence spaces and non-Newtonian calculus, J. Inequal. Appl. 2012, Article ID 932734, 12pp., (2012)

[6] R. Çolak, M. Et, On some generalized difference sequence spaces and related matrix transformations, Hokkaido Math. J. 26, pp. 483-492, (1997).

[7] M. Et, Rifat Çolak, On Some Generalized Difference Sequence Spaces, Soochow J. Math. 21 (4), pp. 377-386, ....

[8] D. J. H. Garling, The $\beta$ - and $\gamma$-duality of sequence spaces, Proc. Camb. Phil. Soc. 63, pp. 963-981, (1967).

[9] M. Grossman, R. Katz, Non-Newtonian Calculus, Lee Press, Piegon Cove, Massachusetts, (1972).

[10] M. Grossman, Bigeometric Calculus: A System with a scale-Free Derivative, Archimedes Foundation, Massachusetts, (1983).

[11] J. Grossman, M. Grossman, R. Katz, The First Systems of Weighted Differential and Integral Calculus, University of Michigan, (1981).

[12] J. Grossman, Meta-Calculus: Differential and Integral, University of Michigan, (1981).

[13] H. Kizmaz, On Certain Sequence Spaces, Canad. Math. Bull. 24 (2), pp. 169-176, (1981).

[14] G. Köthe, Toplitz, Vector Spaces I, Springer-Verlag, (1969).

[15] G. Köthe, O. Toplitz, Linear Raume mit unendlichen koordinaten und Ring unendlichen Matrizen, J. F. Reine u. Angew Math. 171, pp. 193-226, (1934).

[16] I.J. Maddox, Infinite Matrices of Operators, Lecture notes in Mathematics, 786, Springer-Verlag, (1980).

[17] D. Stanley, A multiplicative calculus, Primus IX 4, pp. 310-326, (1999).

[18] S. Tekin, F. Başar, Certain Sequence spaces over the non-Newtonian complex field, Abstr. Appl. Anal. 2013(2013) Article ID 739319, 11 pages.

[19] C. Türkmen, F. Başar, Some Basic Results on the sets of Sequences with Geometric Calculus, Commun. Fac. Fci. Univ. Ank. Series A1. G1(2), pp. 17-34, (2012). 
[20] A. Uzer, Multiplicative type Complex Calculus as an alternative to the classical calculus, Comput. Math. Appl. 60, pp. 2725-2737, (2010).

\section{Khirod Boruah}

Department of Mathematics, Rajiv Gandhi University, Rono Hills, Doimukh-791112, Arunachal Pradesh, India

e-mail : khirodb10@gmail.com

and

\section{Bipan Hazarika}

Department of Mathematics,

Rajiv Gandhi University, Rono Hills, Doimukh-791112, Arunachal Pradesh, India

e-mail : bh_rgu@yahoo.co.in 